\title{
Diseño de aplicación móvil para promoción de productos
}

\author{
Fecha de recepción: 2021-11-27 • Fecha de aceptación: 2022-01-21 • Fecha de publicación: 2022-02-10
}

\author{
Andrea Guissela Pérez Lozada ${ }^{1}$ \\ Graficar Publicidad Gramedia, Ecuador \\ andre_g0397@hotmail.com \\ https://orcid.org/0000-0001-7908-091X \\ Edison Moreno Jurado² \\ SRI - Analista de Seguridad, Ecuador \\ mondeo_eddy33@hotmail.com \\ https://orcid.org/0000-0001-6278-7097 \\ Luis Alberto Pérez Viana ${ }^{3}$ \\ Telconet Latam, Ecuador \\ dark200486@gmail.com \\ https://orcid.org/0000-0002-9844-2274
}

\section{RESUMEN}

La idea de este trabajo surge al realizar un análisis de la necesidad que contemplan los emprendedores para dar a conocer sus productos o servicios. De esta forma se logró plantear el objetivo del proyecto que fue desarrollar un prototipo para dispositivos móviles Android, que permita crear un catálogo de productos. Con base en métodos de inferencia, fuentes bibliográficas y encuestas resumidas sobre las preferencias comerciales de los consumidores y las marcas favoritas, tres aspectos que son importantes para los usuarios cuando compran son: direcciones, precios y ubicación, que son los puntos clave para diseñar una aplicación llamada Deals app, el cual brindará información a los usuarios para que puedan filtrar las compras o servicios de acuerdo a sus necesidades y resolver los inconvenientes que impidan una compra satisfactoria, fomentando así el uso de las nuevas tecnologías que brindan un medio de publicidad para comerciantes y negocios y configuran una plataforma de consulta para los consumidores. 
PALABRAS CLAVE: producto, comercial, móvil, catálogo, asesor de compras

\section{ABSTRACT}

The idea of this work arises from an analysis of the need that entrepreneurs have to publicize their products or services. Thus, the objective of the project was to develop a prototype for Android mobile devices, which allows the creation of a product catalog. Based on inference methods, bibliographic sources and summarized surveys on the commercial preferences of consumers and favorite brands, three aspects that are important for users when shopping are: addresses, prices and location, which are the key points to design an application called Deals app, which will provide information to users so they can filter purchases or services according to their needs and solve the problems that prevent a successful purchase, thus promoting the use of new technologies that provide a means of advertising for merchants and businesses and set up a consultation platform for consumers

KEYWORDS: product, commercial, mobile, catalogue, purchasing advisor 


\section{Introducción}

Hoy en día, la sociedad enfrenta cada vez más necesidades en educación, sociedad, trabajo y otros campos, lo cual ha generado un gran impacto en las personas, experimentando un gran auge tecnológico que los obliga a capacitarse. En un mundo cada vez más competitivo, las herramientas tecnológicas se han convertido en imprescindibles para mejorar y perfeccionar los procesos de trabajo y comunicación (Piernas, 2014).

Surgen preocupaciones entonces, ¿cuáles son los factores influyentes que pueden conducir a un consumo más eficiente? Las marcas de productos luchan por establecerse en la mente de los consumidores, especialmente de los usuarios de Internet, que tienen una presencia activa en el mercado. Hoy en día hay un crecimiento exponencial de marketing por redes sociales y aplicaciones móviles, el uso de estas ha crecido durante la pandemia (Villacís et al., 2010).

Actualmente, en la literatura existen trabajos relacionados al desarrollo de aplicaciones móviles que solventan problemas cotidianos, aplicaciones para el teletrabajo (Reyes et al., 2020), para registro y búsqueda de mascotas (Burbano et al., 2020), para servicio de taxis (Artieda et al., 2021), turismo (Silva et al., 2018), entre otros, lo que es una base para el desarrollo de nuevas soluciones mediante aplicaciones móviles

En este contexto, las marcas de productos buscan expandir su presencia en línea al llegar a más personas a través de tantos canales en línea como sea posible, como las redes sociales y las aplicaciones, siempre que mantengan el código de la empresa.

Para protegerlos, surge la idea de un modelo de aplicación móvil que permita a los usuarios consultar, registrar y procesar información de productos en tiempo real en cualquier lugar, y les permita mejorar sus compras de zapatos y accesorios, ropa y consumibles, equipos, servicios, brindando información de acuerdo a las necesidades del usuario, ya sea direcciones, precios y/o ubicaciones.

\section{Metodología}

El presente proyecto tiene como distintivo el desarrollo de un catálogo como medio viable, incorporando funciones que facilite la publicidad de productos ofertados por comerciales y empresas del Ecuador.

Por lo tanto, se utiliza el método deductivo (Prieto, 2017) en base a las siguientes premisas:

- Los sistemas informáticos permiten automatizar procesos.

- Este proyecto propone el desarrollo de un sistema informático.

- El sistema propuesto automatizara el proceso de publicidad. 
Se ha desarrollado una aplicación para dispositivos móviles Android (Verborgh et al., 2013), utilizando servicios web y el formato JSON para interpretar los datos desde una fuente disponible en una base de datos externa.

\subsection{Arquitectura de la aplicación}

La arquitectura definida en el desarrollo va a ser hibrida, tipo Modelo, Vista, Controlador (MVC) (Díaz \& Fernández, 2012), para el teléfono (Ver Figura 1), por capas para el modelado de datos (Vásquez, 2010) (Ver Figura 2).

Figura 1.

Arquitectura de la aplicación Móvil

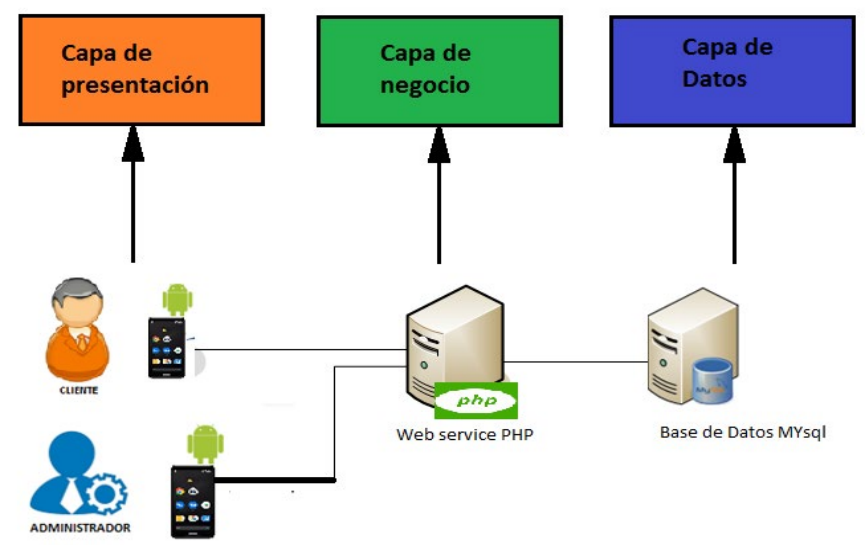

Figura 2.

Arquitectura por capas
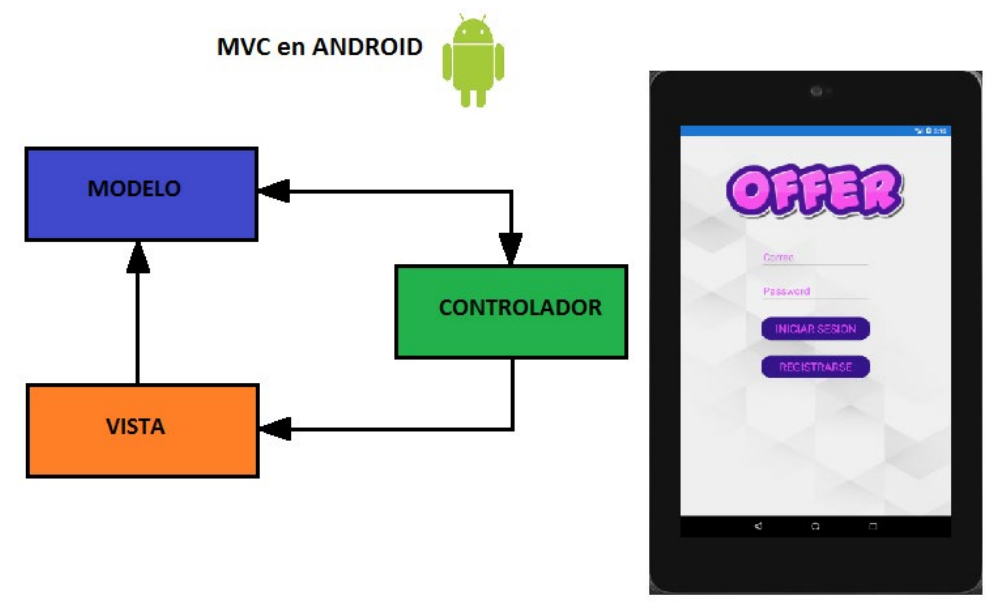

Una vez que estén funcionando adecuadamente todos los servicios de la aplicación propuesta se tiene una arquitectura final descrita en la Figura 3 y Figura 4. 
Figura 3.

Arquitectura sistema cliente

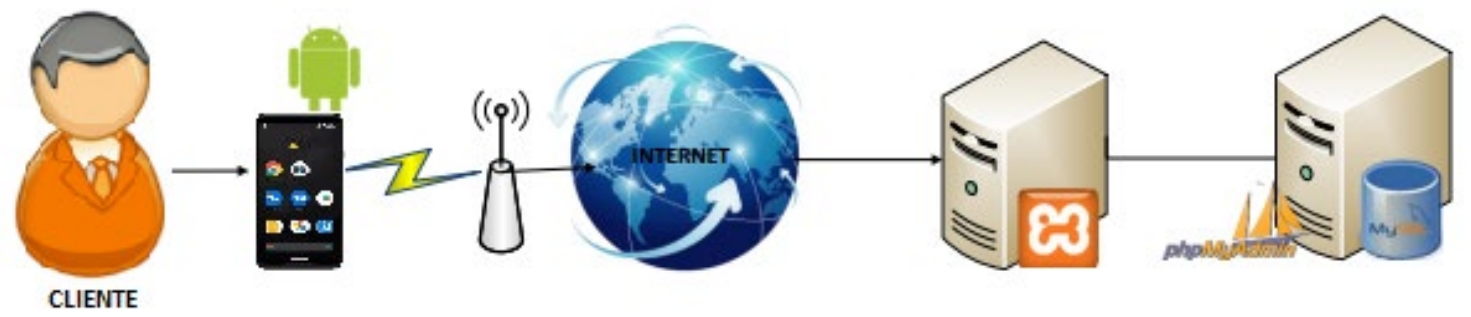

Figura 4.

Arquitectura del sistema administrativo
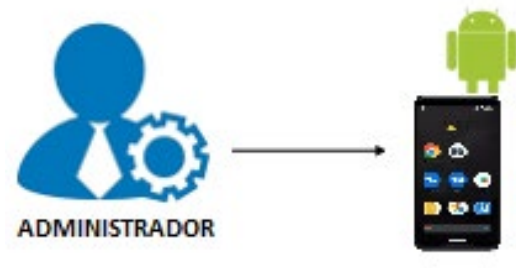

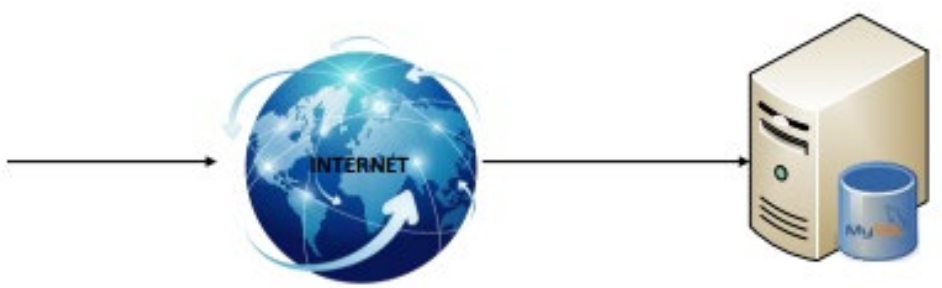

Fuente: Modelado Paint

\subsection{Plataformas de desarrollo}

Actualmente existen distintas plataformas de desarrollo móvil de tipo nativas, hibridas y multiplataforma, en este trabajo se optó por utilizar Xamarin (s.f). En la Tabla 1 se detalla las herramientas utilizadas.

\section{Tabla 1.}

Descripción de Herramientas Tecnológicas

\begin{tabular}{ll}
\hline Herramienta & Descripción \\
\hline XAMARIN & Framework de desarrollo móvil multiplataforma \\
\hline XAMARIN FORMS & Permite que la APP, sea multiplataforma \\
\hline MY SQL & Motor de Base de Datos \\
\hline VISUAL STUDIO.NET & MS Visual Studio 2019. Framework 4.8 \\
\hline REST & Servicios Web \\
\hline
\end{tabular}




\subsection{Flujo en el proceso de aplicación móvil}

La Figura 5 detalla el flujo de acciones que se van a desarrollar en el dispositivo móvil y la Figura 6 sobre el proceso del administrador.

Figura 5.

Proceso cliente

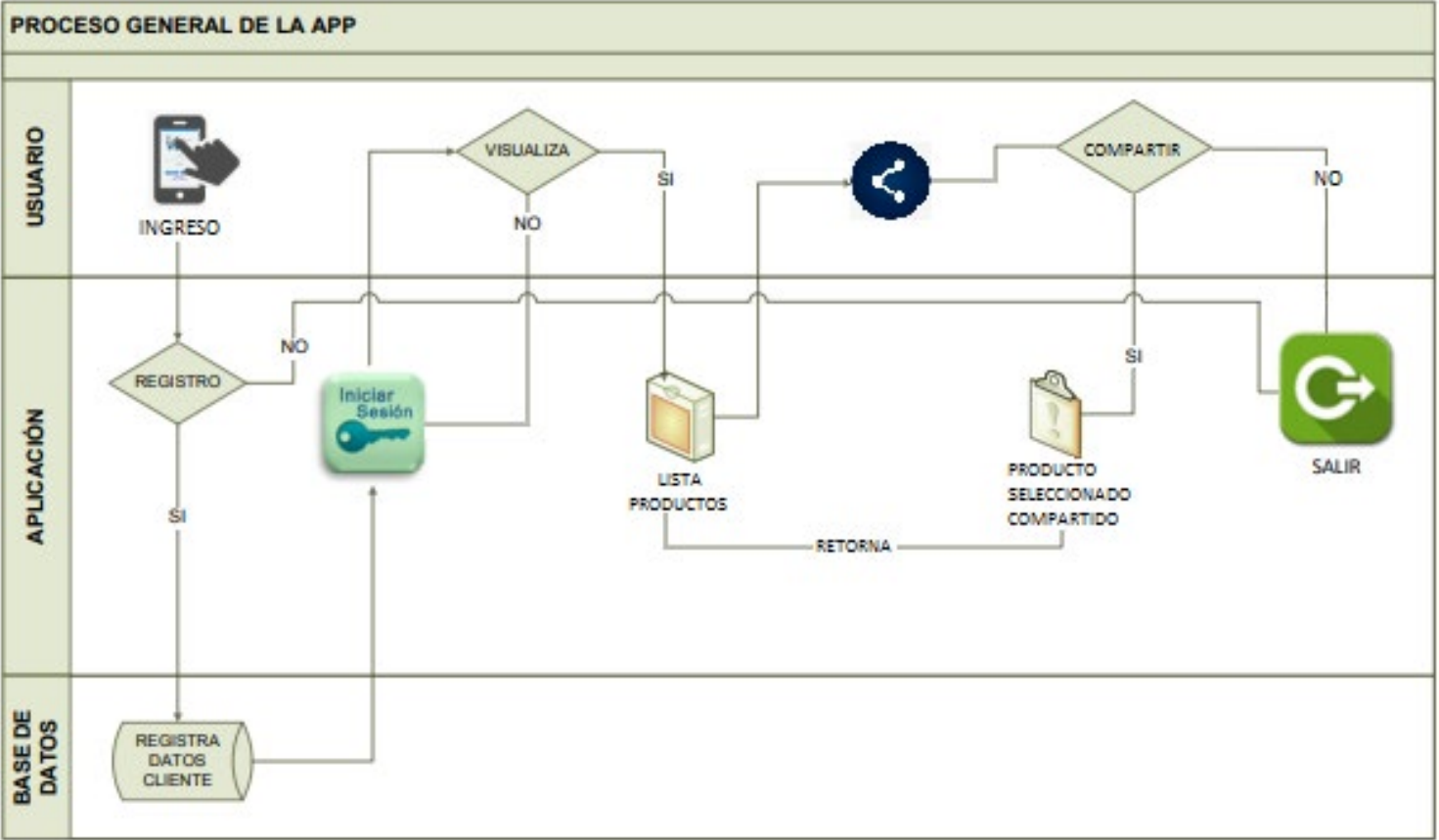

Fuente: Modelado End to End 
Figura 6.

\section{Proceso administrador}

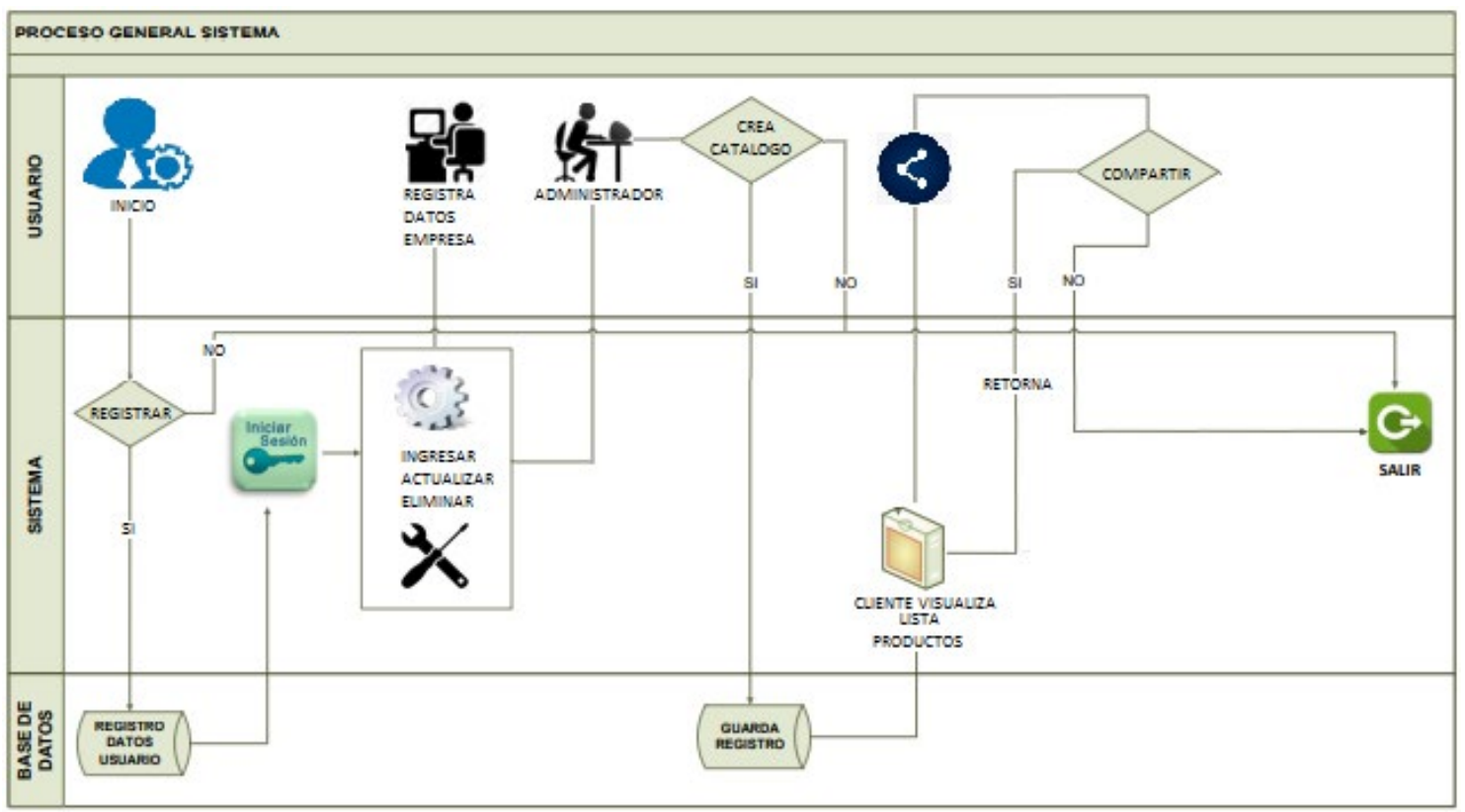

Fuente: Modelado End to End

\subsection{Requerimientos}

En el desarrollo de aplicaciones informáticas es necesario definir los requerimientos que se deben cumplir, en este sentido la Tabla 2 y Tabla 3 describen los requerimientos funcionales y no funcionales.

Tabla 2.

Requerimientos funcionales

\begin{tabular}{ll}
\hline \multicolumn{2}{l}{ Requerimientos Funcionales } \\
\hline$R F-01$ & Se permitirá registrar la información de usuarios y los datos de su comercial o empresa. \\
\hline$R F-02$ & Acceso por usuario y contraseña \\
\hline$R F-03$ & Creación de catálogo de productos. \\
\hline$R F-04$ & Se permitirá listar, filtrar la cartera de productos categorizados considerando. \\
\hline$R F-05$ & El sistema permitirá insertar, editar, eliminar, exportar archivos del catálogo. \\
\hline$R F-06$ & El sistema permitirá anexar redes sociales para compartir archivos. \\
\hline
\end{tabular}


Tabla 3.

\section{Requerimientos No Funcionales}

\begin{tabular}{ll}
\hline Requerimientos No Funcionales \\
\hline RNF - 01 & $\begin{array}{l}\text { Se configurará para que funcione correctamente en la franja más vasta viable de } \\
\text { dispositivos Android, desde su versión más baja. }\end{array}$ \\
\hline RNF - 02 & Inicialmente funcionara en Android, posterior para iOS \\
\hline RNF - 03 & La aplicación proporcionará mensajes de ayuda, advertencias y errores. \\
\hline RNF - 04 & El acceso los datos por roles de usuario. \\
\hline RNF - 05 & El sistema tendrá robustez para garantizar que la aplicación esté siempre disponible. \\
\hline RNF - 06 & El sistema se concentrará en el uso intuitivo (facilidad de uso). \\
\hline
\end{tabular}

\subsection{Diagrama UML}

Para representar los esquemas del programa se utiliza herramientas de modelado UML (Ahmad et al., 2019), tal cual se obtuvo una abstracción de las propiedades primordiales de la aplicación, en la Figura 7 se grafica.

\section{Figura 7.}

Diagrama UML
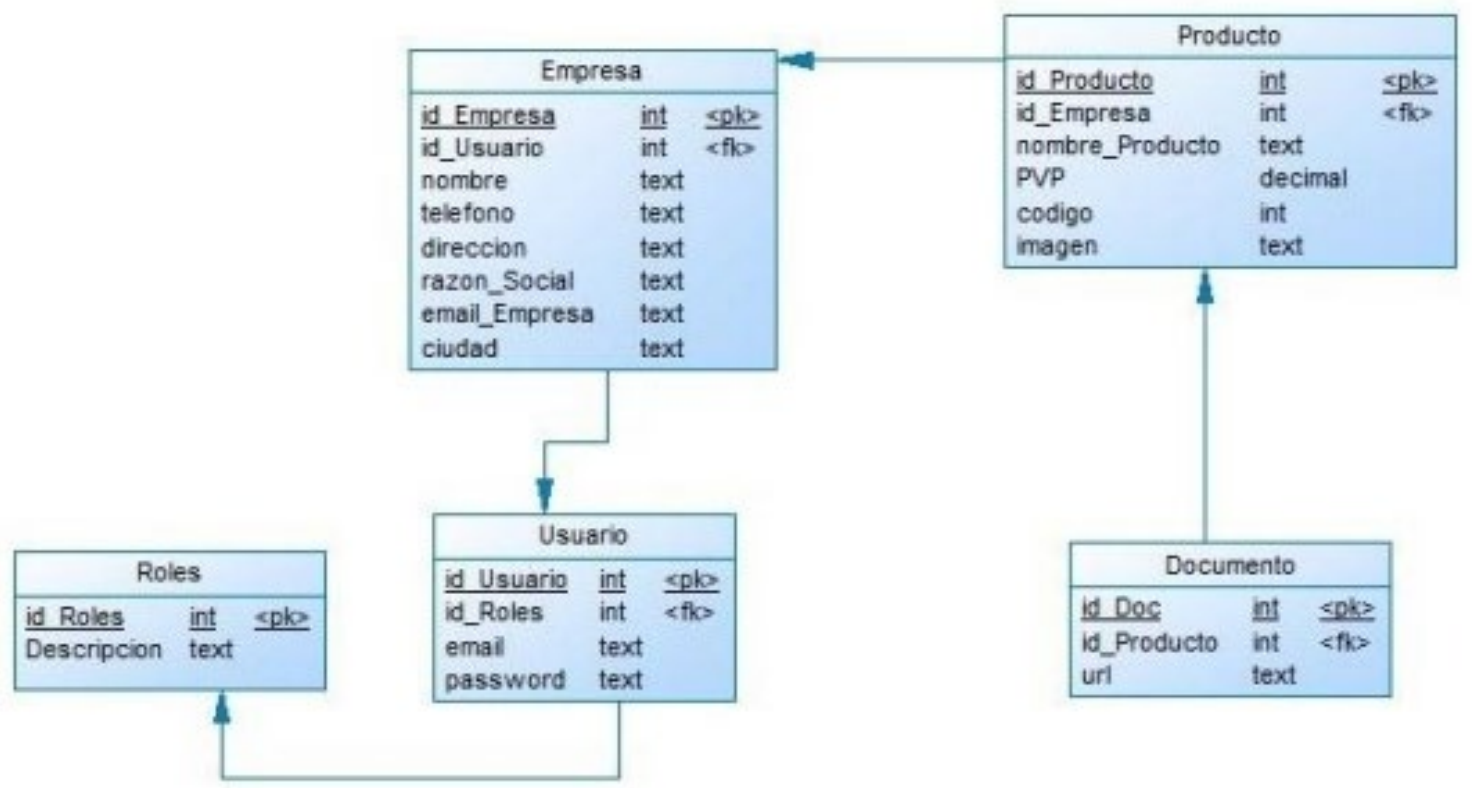

Fuente: Power Designer 


\subsection{Caso de uso}

\section{La Figura 8 muestra la relación de actores con la aplicación y el sistema.}

\section{Figura 8.}

Caso de uso

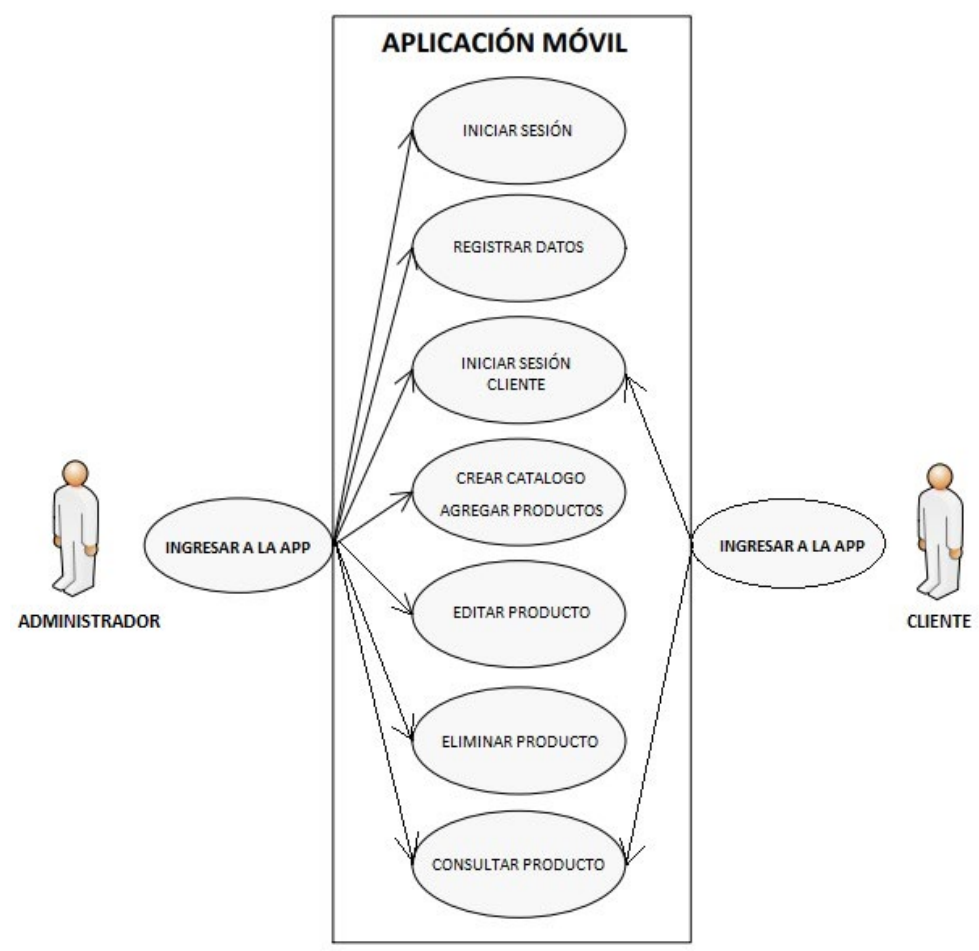

La Tabla 4 y 5 detallan los casos de uso de cada actor, es decir para el administrador y los usuarios

Tabla 4.

Descripción de diagrama caso de uso administrador

\begin{tabular}{ll}
\hline Caso de uso & Diagrama de la aplicación \\
\hline Actor & Vendedor \\
\hline Descripción & $\begin{array}{l}\text { El vendedor debe iniciar sesión para registrar los datos de su empresa y realizar } \\
\text { acciones como ingresar un producto, modificar, eliminar, vía aplicación móvil. }\end{array}$ \\
\hline Pre-condición & Acceso a internet \\
\hline Actividades & Ingreso al sistema con usuario y contraseña. \\
\cline { 2 - 2 } & Presentación lista de catálogo de productos con imagen y precio. \\
\cline { 2 - 2 } & Insertar datos: cargar datos de productos. \\
\cline { 2 - 2 } & Modificar datos: editar datos de productos. \\
\cline { 2 - 2 } & Eliminar datos: eliminar datos de productos. \\
\cline { 2 - 2 } & Compartir catálogo: Compartir catálogo por medio de redes sociales. \\
\hline
\end{tabular}


Tabla 5.

Descripción de diagrama caso de uso cliente

\begin{tabular}{ll}
\hline Caso de Uso & Diagrama de la aplicación \\
\hline Actor & Usuario \\
\hline Descripción & Debe registrar sus datos para iniciar sesión y acceder al sistema \\
\hline Pre-condición & Acceso a internet \\
\hline Actividades & Acceso al sistema por usuario y contraseña \\
\cline { 2 - 2 } & Presentación lista de catálogo de productos con imagen y precio. \\
\cline { 2 - 2 } & Compartir catálogo: Compartir catálogo por medio de redes sociales. \\
\hline Flujo alternativo & Control de intentos de acceso al sistema. \\
\hline
\end{tabular}

\subsection{Diagrama de base de datos}

Al ser un sistema pequeño, se utilizan 5 tablas relacionadas para el flujo de los datos, la Figura 9 muestra el modelo entidad relación de la base de datos. En este trabajo se utilizó el gestor de base de datos MYSQL (s.f).

\section{Figura 9.}

Base de datos

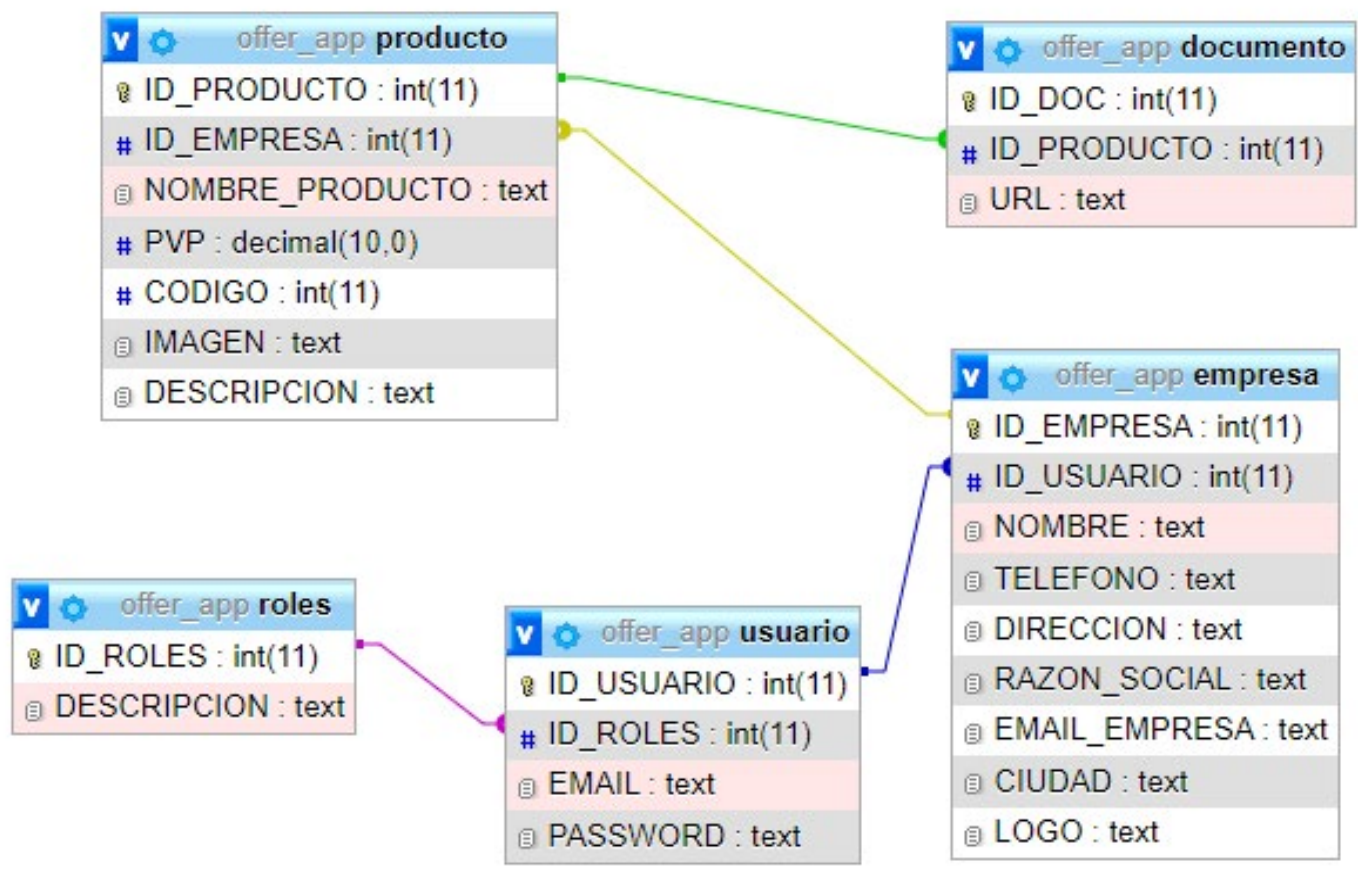




\subsection{Prototipado}

A continuación, se presenta el diseño preliminar de las pantallas en la herramienta Balsamiq Mockups (Balsamiq, s.f). La Figura 10 muestra las pantallas iniciales del sistema definidas en la fase re recolección de información.

Figura 10.

Prototipado

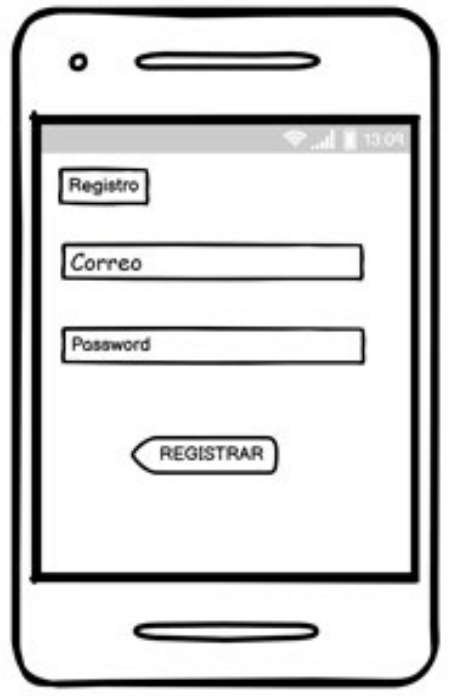

Visto preliminar App Ingreso

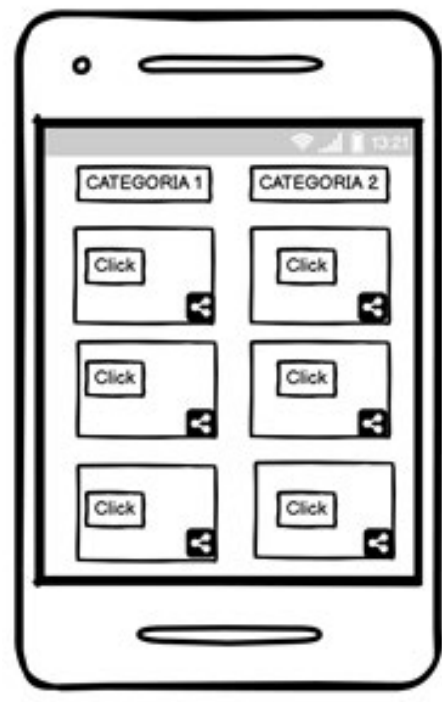

Visto preliminor App experiencia de uso

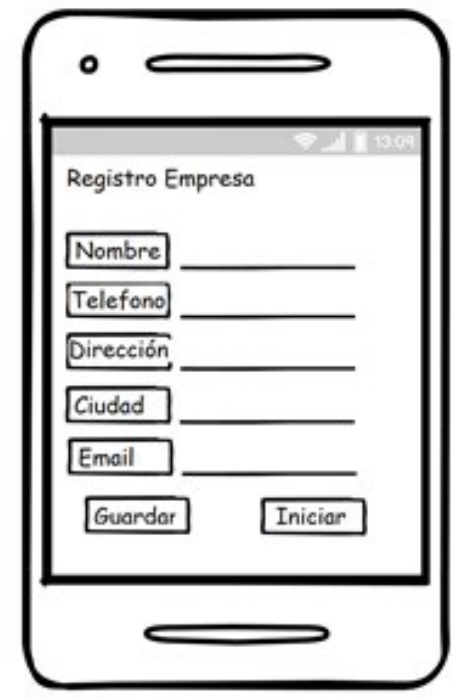

Visto preliminer RDatos

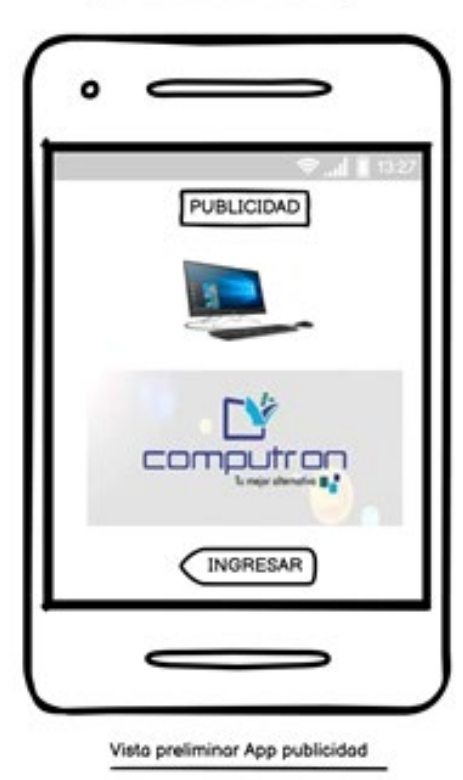

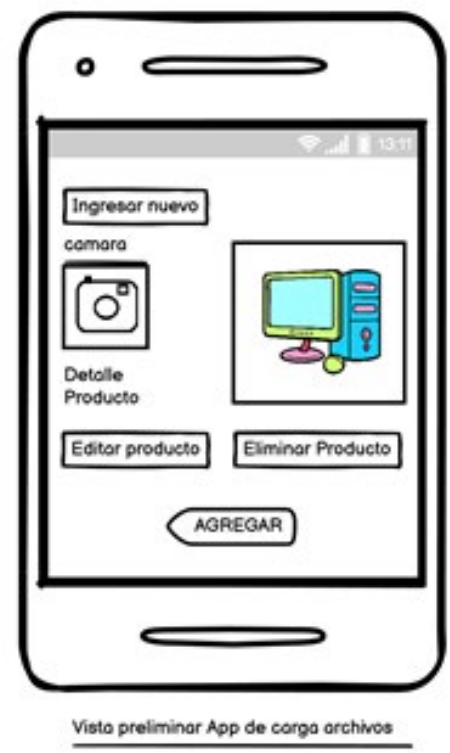

Visto preliminar App de corga archwos 


\subsection{Descripción interfaz de la App}

La pantalla inicial del sistema tiene 2 botones comenzar sesión, registrarse y en la parte preeminente se muestra el logo de la aplicación (Ver Figura 11).

Figura 11.

Descripción Interfaz
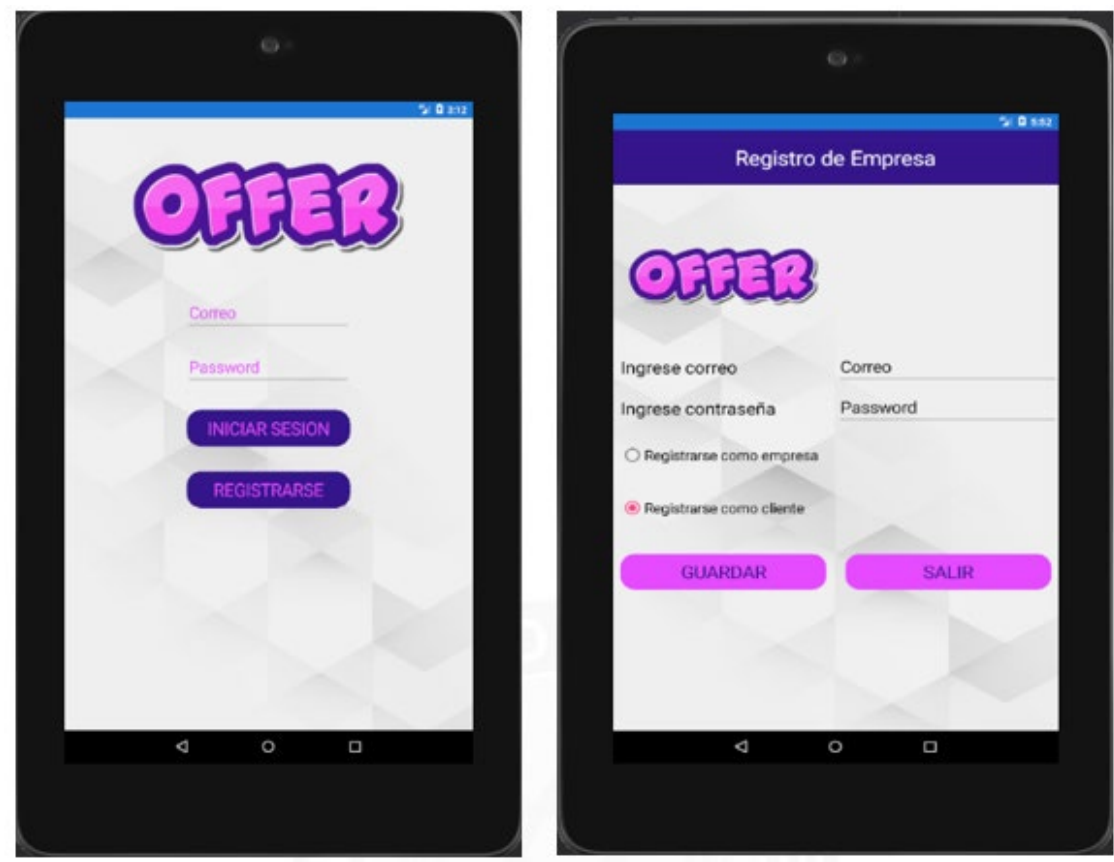

Una vez que el usuario accede al sistema, tiene la opción de registrar su empresa para poder promocionarla, esto se muestra en la Figura 12. 
Figura 12.

Registrar, Empresa
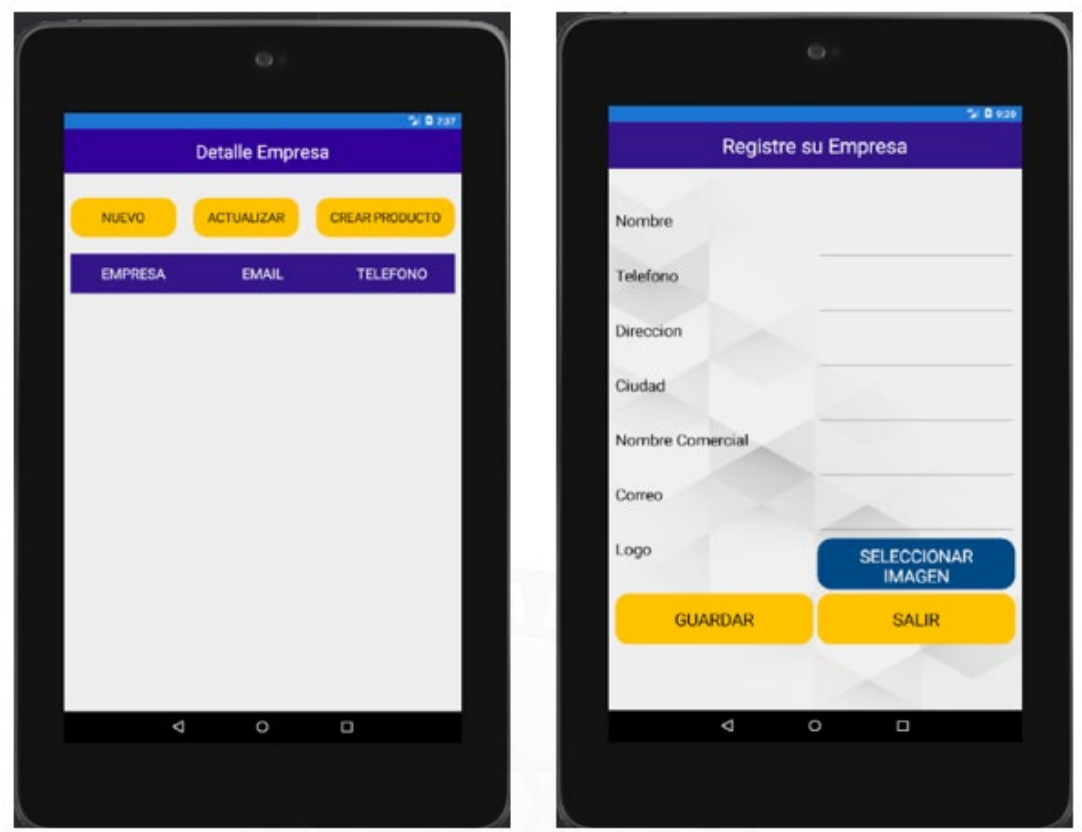

Cuando se registra la empresa, aparece en el listado de empresas que están promocionándose, ver la Figura 13.

Figura 13.

Crear, Actualizar, Eliminar Catalogo
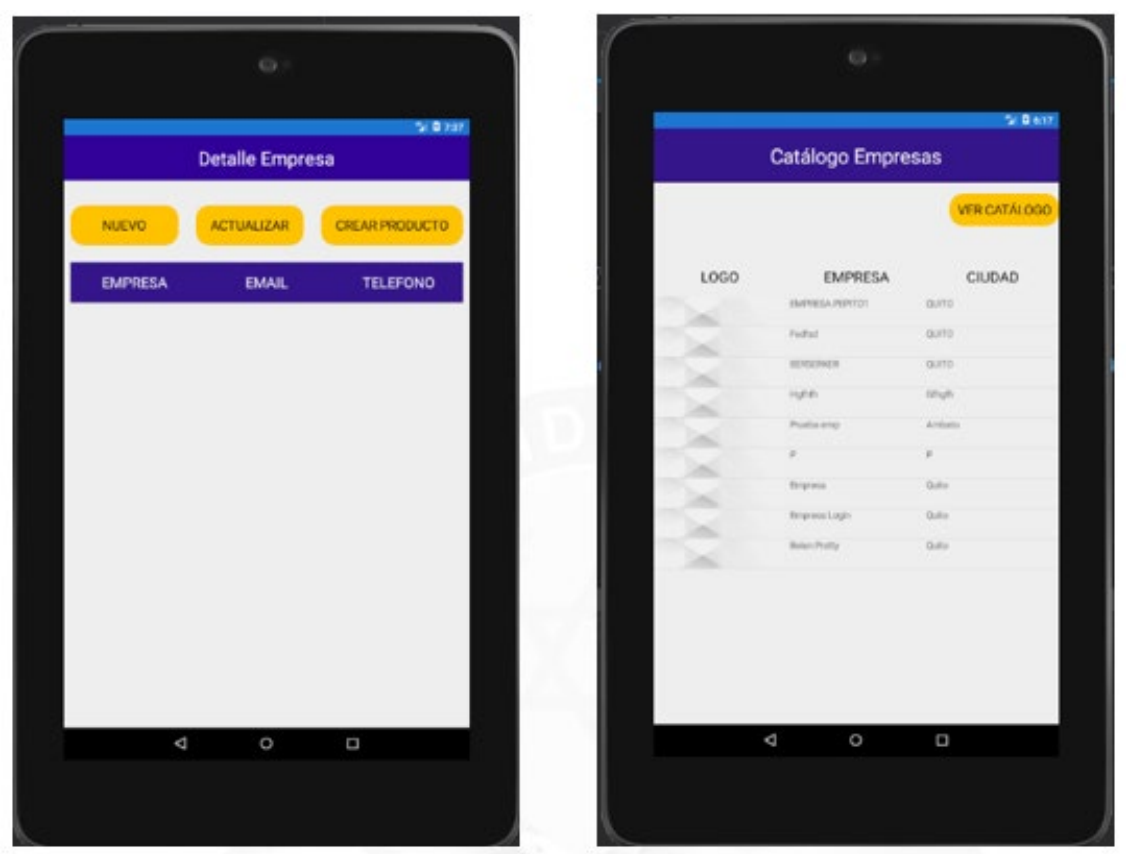
Finalmente, en la Figura 14 se muestran los detalles de la empresa y el producto que promocionan.

Figura 14.

Ver Catalogo, Seleccionar un Producto
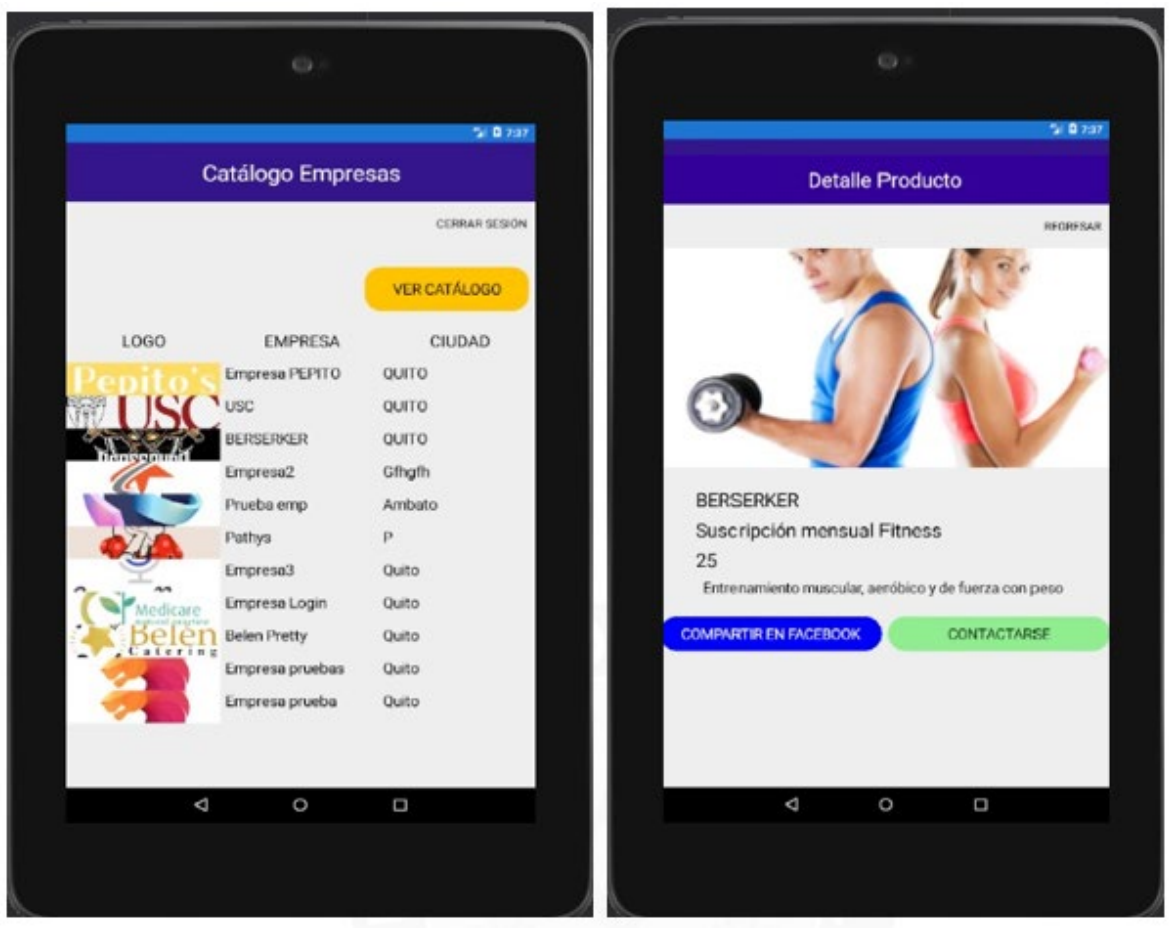

\section{Resultados}

Una vez finalizada la fase de codificación, se realizaron las pruebas respectivas, tomando en cuenta los aspectos siguientes:

- Síntesis sobre los comentarios de usuarios al momento de utilizar la aplicación, tomando en cuenta una autenticación correcta y una autenticación incorrecta.

- Comprobación funcional en diferentes teléfonos con requisitos mínimos para la instalación de la aplicación.

A continuación, se muestra un test que tiene como objetivo evaluar la percepción del usuario al usar la aplicación Offer App y analizar 5 puntos críticos que determinarán:

Tabla 6.

Criterios a evaluar

Criterios de evaluación

Aspecto Descripción 


\begin{tabular}{ll}
\hline Contenido & De acuerdo a la normativa definida por la empresa \\
\hline $\begin{array}{l}\text { Facilidad de } \\
\text { Aprendizaje }\end{array}$ & Resultados al usar la aplicación por primera vez. \\
\hline Accesibilidad & Consideraciones Visuales \\
\hline Satisfacción & Grado de Satisfacción del usuario \\
\hline Eficiencia & Adaptación de usuarios frente al uso de la aplicación . \\
\hline
\end{tabular}

Tabla 7.

Prueba autenticación

\begin{tabular}{ll}
\hline Pruebas de Usabilidad \\
\hline Descripción & $\begin{array}{l}\text { Una vez instalada la aplicación aparece una ventana de "Inicio de Sesión”, en el que ingresa } \\
\text { su correo y contraseña internamente se validan estos datos. }\end{array}$ \\
\hline $\begin{array}{l}\text { Condiciones de eje- } \\
\text { cución }\end{array}$ & El usuario debe estar registrado en el sistema \\
\hline Entrada & $\begin{array}{l}\text { El usuario cliente ingresa su correo, contraseña y presiona "Iniciar Sesión" ingresa a venta- } \\
\text { na, ver catálogos. }\end{array}$ \\
\cline { 2 - 2 } & $\begin{array}{l}\text { El usuario administrador presiona "Registrarse" ingresa a ventana de Registro Administra- } \\
\text { dor e ingresa su correo, contraseña para registro. }\end{array}$ \\
\hline Resultado Esperado & $\begin{array}{l}\text { Tras el ingreso de correo y contraseña, si el proceso ha sido correcto en la base de datos se } \\
\text { registra y el usuario puede utilizar la aplicación. }\end{array}$ \\
\hline $\begin{array}{l}\text { Evaluación de la } \\
\text { prueba }\end{array}$ & \begin{tabular}{l} 
Prueba satisfactoria. \\
\hline
\end{tabular}
\end{tabular}

- Participantes: se tomará una muestra de 20 personas negociantes. La prueba de usabilidad se llevará a cabo bajo la supervisión de los desarrolladores.

- Criterio de aceptación o rechazo:

- Errores leves: confusión del usuario con algún botón

- Errores medios: falta de personalización para presentar datos.

- Errores críticos: la sincronización no garantiza que la información de la base de datos sea igual a la del dispositivo móvil, se almacena información errónea con inconsistencia de datos, el proceso de actualización no es satisfactorio.

\section{Conclusiones}

Luego de hacer toda la investigación pertinente de la App, se puede deducir que los individuos permanecen abiertas al uso del Internet en dispositivos móviles y las novedosas tecnologías, así sea por gusto o por necesidad, por ende, el brindar una aplicación móvil, con propiedades diferenciadoras como tiempos y movimientos, que son recursos principales en la actualidad, dichos realizan de Offer App una aplicación distinta en un sector de veloz incremento y con alta competitividad. 
Offer App se convierte en un plan innovador de apoyo publicitario, al comerciante, al empresario y al consumidor, debido a que es algo con lo cual el desarrollo del mercado del Ecuador necesita para asegurar su aumento.

Referente a la App tiene 2 instancias relevantes, la primera es que al inicio va a ser gratuita y la segunda su diseño e implementación va a ser agradable, simple de usar debido a las tácticas de la arquitectura planteada.

Uno de los enfoques con más auge en los últimos años es el marketing digital, el cual permitió que las empresas potencien su marca usando canales de repartición como son las redes sociales, por ende, Offer App va conforme a estas novedosas tendencias en el desarrollo de herramientas tecnológicas. 


\section{Referencias}

Ahmad, T., lqbal, J., Ashraf, A., Truscan, D., \& Porres, I. (2019). Model-based testing using UML activity diagrams: A systematic mapping study. Computer Science Review, 33, 98-112. https://doi.org/10.1016/j.cosrev.2019.07.001

Artieda, C., Reza, J., \& Rojas, H. (2021). Sistema móvil para la oferta de recorridos con rutas fijas. REVISTA ODIGOS, 2(1), 21-40. https://doi.org/10.35290/ro.v2n1.2021.381

Balsamiq. (s.f). Rapid, Effective and Fun Wireframing Software. https://balsamiq.com/

Burbano, E., Constante, M., Hidalgo, L., \& Moya, F. (2020). Prototipo móvil para la geolocalización de mascotas callejeras. REVISTA ODIGOS, 1(3), 77-96. https://doi.org/10.35290/ro.v1n3.2020.372

Díaz, Y., \& Fernández, Y. (2012). Patrón Modelo-Vista-Controlador. Telemática, 11(2). https://www.revistatelematica.cujae.edu.cu/index.php/tele/article/view/15

MySQL. (s.f). MySQL 5.7 Reference Manual : 1.3.1 What is MySQL? https://dev.mysql.com/doc/refman/5.7/en/ what-is-mysql.html

Piernas, R. (24 de abril de 2014). ¿Qué es el Engagement en las Redes Sociales? Bilnea. https://bilnea.com/ que-es-engagement-en-redes-sociales/

Prieto, B. (2017). El uso de los métodos deductivo e inductivo para aumentar la eficiencia del procesamiento de adquisición de evidencias digitales. Cuadernos de contabilidad, 18(46), 56-82. https://doi.org/10.11144/ javeriana.cc18-46.umdi

Reyes, A., Vallejos, M. de L., \& Quintana, D. (2020). Nueva realidad: compras en línea y a domicilio. REVISTA ODIGOS, 1(3), 47-60. https://doi.org/10.35290/ro.v1n3.2020.370

Silva, C. A., Toasa, R., Guevara, J., Martinez, H. D., \& Vargas, J. (2018). Mobile Application to Encourage Local Tourism with Context-Aware Computing. In 2018 International Conference on Information Technology \& Systems, ICITS'18, 796-803. https://doi.org/10.1007/978-3-319-73450-7 75

Vásquez, F. A. (2010). Programación por capas. Paradigmas, 2(1), 13-29.

Verborgh, R., Harth, A., Maleshkova, M., Stadtmüller, S., Steiner, T., Taheriyan, M., \& Van de Walle, R. (2014). Survey of semantic description of REST APIs. In REST: Advanced Research Topics and Practical Applications (pp. 69-89). Springer. https://doi.org/10.1007/978-1-4614-9299-3 5 
Villacís, B., Carrillo, D., \& Martínez, A. (2010). Estadística demográfica en el Ecuador: diagnóstico y propuesta. Instituto Nacional de Estadística y Censos, 1-74. https://www.ecuadorencifras.gob.ec/wp-content/descargas/Libros/Demografia/documentofinal1.pdf

Xamarin. (s.f). What is Xamarin? - I Microsoft Docs. 
Copyright (c) 2022 Andrea Guissela Pérez Lozada, Edison Moreno Jurado y Luis Alberto Pérez Viana

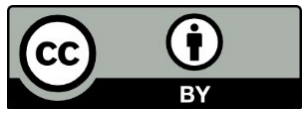

Este texto está protegido bajo una licencia internacional Creative Commons 4.0.

Usted es libre para Compartir-copiar y redistribuir el material en cualquier medio o formato - y Adaptar el documento - remezclar, transformar y crear a partir del material-para cualquier propósito, incluso para fines comerciales, siempre que cumpla las condiciones de Atribución. Usted debe dar crédito a la obra original de manera adecuada, proporcionar un enlace a la licencia, e indicar si se han realizado cambios. Puede hacerlo en cualquier forma razonable, pero no de forma tal que sugiera que tiene el apoyo del licenciante o lo recibe por el uso que hace de la obra.

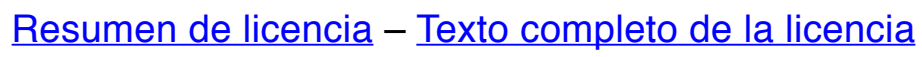

